\title{
Model networks of end-linked polydimethylsiloxane chains. I. Comparisons between experimental and theoretical values of the elastic modulus and the equilibrium degree of swelling
}

\author{
J. E. Mark and J. L. Sullivan \\ Department of Chemistry and the Macromolecular Research Center, University of Michigan, Ann Arbor, \\ Michigan 48109 \\ (Received 20 September 1976)

\begin{abstract}
Two samples of hydroxyl-terminated polydimethylsiloxane having molecular weights the order of $2 \times 10^{4}$ and $1 \times 10^{3} \mathrm{~g} \mathrm{~mol}^{-1}$ were separated into a total of six fractions of relatively narrow molecular weight distribution. Portions of one of the unfractionated polymers and each of the fractions were cross-linked by reacting the hydroxyl chain ends, in the undiluted state, with a tetrafunctional orthosilicate. The resulting networks of end-linked chains were studied with regard to their stress-strain isotherms in elongation at $25^{\circ} \mathrm{C}$ and their equilibrium swelling in benzene at room temperature. Values of the elastic modulus obtained from the isotherms support theoretical arguments that fluctuations in the network chain vectors reduce the value of the modulus to approximately one-half of the value predicted for affine deformations of chain vectors constrained in their fluctuations by cross-links firmly embedded in the network medium. Values of the equilibrium degree of swelling of the networks calculated on this basis are also in good agreement with experiment. The networks formed by end-linking relatively short chains have small values of the semiempirical constant $2 C_{2}$ used as a measure of the departure of an observed stress-strain isotherm from the form predicted by theory. Although this observation is consistent with the suggestion that such end-linked networks have a much smaller number of interchain entanglements than do randomly cross-linked networks, other evidence and arguments unfortunately do not support this assumption.
\end{abstract}

\section{INTRODUCTION}

Elastomeric polymer networks are generally prepared from chain molecules of very high molecular weight, by chemically joining or "cross-linking" chain units in a highly random fashion. " The cross-linking techniques most widely used at the present time, for example, are sulfur vulcanization, peroxide curing, and $\gamma$-irradiation, and all fall into this category. Unfortunately, a network formed in this way has a highly complex structure in that there is generally a relatively broad distribution of network chain lengths, a significant fraction of imperfections such as dangling chain ends, and presumably some entanglements between neighboring chains which act to some extent as physical cross-links. ${ }^{1-4}$ For this reason, there has been a great deal of interest in the preparation of polymer networks using techniques which permit better control over the cross-linking process and thus over the structure of the resulting network. ${ }^{5-18}$ It would obviously be highly desirable to be able to introduce cross-links in a less random manner so that knowledge of the crosslinking mechanism would permit an independent estimate of the cross-link density in the network, and thus of the average length of the network chains.

of the new synthetic techniques being developed for this purpose, those showing the greatest promise involve the joining of polymer chains exclusively at their ends. In highly selective reactions of this type, functional groups placed at the two ends of each of the chains are chemically reacted with cross-linking agents having a functionality 3 or greater. If each chain is successfully reacted with two different cross-linking sites, the result is an "ideal," "tailor-made," or "model" network in that the network chains would have the same average length and distribution of lengths as the sam- ple of non-cross-linked chains from which it was prepared. This direct and independent measure of the average chain length would be of great importance in the evaluation of the molecular theories of rubberlike elasticity,$^{1-4,17-23}$ in which there is some uncertainty in the numerical factor relating the elastic modulus of the network to the molecular weight of the network chains. $9,10,22$ Also of importance is the fact that it is thus now possible to prepare networks of relatively uniform chain length by using these end-linking techniques on polymers known to be nearly monodisperse by virtue of their having been polymerized with a suitable anionic initiator, or on polymer fractions obtained using well-known separation techniques which give materials of relatively narrow molecular weight distribution. Such networks would obviously be useful in characterizing the effect of chain length distribution on the elastomeric properties of a network. Interest in networks of this type has been further enhanced by the suggestion ${ }^{6,16}$ that they may be essentially free of chain entanglements. If end-linking chain molecules in this way were indeed somehow to give polymer networks of such a simplified topology, the study of their elastomeric properties would give definitive answers to important questions such as the relative degree of entangling of randomly cross-linked networks and the extent to which chain entanglements act as physical cross-links in different types of network deformation.

The present investigation involves the preparation of highly elastomeric networks formed by end-linking hydroxyl-terminated polydimethylsiloxane (PDMS) chains of known molecular weight and molecular weight distribution with a tetrafunctional cross-linking agent. Stress-strain isotherms measured on the networks in elongation are used to obtain values of the elastic 
TABLE I. Viscosity-average, weight-average, and numberaverage molecular weights of the non-cross-linked PDMS chains.

\begin{tabular}{llllll}
\hline & \multicolumn{1}{c}{$10^{-3} M_{v}\left(\mathrm{~g} \mathrm{~mol}^{-1}\right)$} & & \\
Sample & Direct $^{\mathrm{a}}$ & $\mathrm{GPC}^{\mathrm{b}}$ & $\left(\mathrm{g} \mathrm{mol}^{-1}\right)$ & $\begin{array}{l}10^{-3} M_{n}^{b} \\
\left(\mathrm{~g} \mathrm{~mol}^{-1}\right)\end{array}$ & $M_{w} / M_{n}$ \\
\hline$A$ & 31.5 & 31.5 & 34.6 & 18.5 & 1.87 \\
$A-1$ & 68.9 & 57.6 & 60.2 & 45.0 & 1.34 \\
$A-2$ & 44.0 & 38.1 & 39.3 & 32.9 & 1.19 \\
$A-3$ & 31.2 & 31.0 & 32.3 & 25.6 & 1.26 \\
$A-4$ & 17.4 & 17.1 & 18.4 & 9.5 & 1.93 \\
$B$ & 3.2 & 2.2 & 2.5 & 1.1 & 2.23 \\
$B-1$ & 9.1 & 7.9 & 8.9 & 4.7 & 1.88 \\
$B-2$ & 5.0 & 4.9 & 5.1 & 4.0 & 1.29 \\
\hline \hline
\end{tabular}

${ }^{a}$ Measured in toluene at $20^{\circ} \mathrm{C}$.

${ }^{\mathrm{b}} \mathrm{Gel}$ permeation chromatography, in toluene at room temperature.

modulus suitable for compa rison with results predicted from the molecular theories of rubberlike elasticity. Swelling equilibrium measurements carried out on the same networks provide an additional test of theory. Also investigated are values of the semiempirical Mooney-Rivlin constant $2 C_{2},{ }^{24}$ which measures the departure of an observed stress-strain isotherm in elongation from the form predicted by the present molecular theories. ${ }^{25}$ All of the experimental results are carefully scrutinized for possible effects of chain length distribution on elastomeric properties and for evidence relevant to the degree of entangling in networks of endlinked chains.

\section{EXPERIMENTAL}

\section{Polymer fractionation}

The polymers used in this investigation were two samples of hydroxyl-terminated PDMS, hereafter designated $A$ and $B$, described as having molecular weights the order of $2 \times 10^{4}$ and $1 \times 10^{3} \mathrm{~g} \mathrm{~mol}^{-1}$, respectively. ${ }^{26}$ Standard fractional precipitation techniques ${ }^{1}$ carried out at $25^{\circ} \mathrm{C}$ were used to separate these two polymers into several fractions. Toluene was chosen as the solvent and acetonitrile as the nonsolvent. (Methanol, which is widely used as the nonsolvent in polymer fractionations, cannot be used with polymer chains of this type, which have potentially reactive end-groups). Initial polymer concentrations were $1 w t \%$ in the case of sample $A$ and $5 \mathrm{wt} \%$ in the case of sample $B$. Four fractions $(A-1$ through $A-4)$ were obtained from sample $A$, and two ( $B-1$ and $B-2)$ from sample $B$; each represented roughly $20 \%$ of the polymer from which it was obtained.

\section{Characterization of the non-cross-linked chains}

Viscosity measurements were carried out on samples $A$ and $B$ and on the fractions de rived therefrom, in toluene at $20^{\circ} \mathrm{C}$, using Cannon-Ubbelohde viscometers. Values of the intrinsic viscosity $[\eta]$ thus determined were interpreted according to the relationship $^{27}$

$$
[\eta]=2.0 \times 10^{-4} M_{v}^{0.86}
$$

also obtained using toluene at $20^{\circ} \mathrm{C}$ and established for PDMS chains having molecular weights in the range $3 \times 10^{3}$ to $2 \times 10^{5} \mathrm{~g} \mathrm{~mol}^{-1}$. Values of the viscosity-average molecular weight $M_{\nu}$ thus calculated for samples $A$ and $B$ and for the six fractions are presented in the second column of Table $I$.

Gel permeation chromatography (GPC) was used to obtain a measure of the breadth of the molecular weight distributions of the same eight polymers. The measurements were carried out at room temperature on a Waters GPC apparatus utilizing five Styragel columns having nominal porosities of $10^{6}, 10^{5}, 10^{4}, 10^{3}$, and $2 \times 10^{2} \AA$, respectively. Toluene was employed as the solvent, and the flow rate was approximately $1 \mathrm{ml} \mathrm{min} \mathrm{m}^{-1}$. Several standard PDMS samples ${ }^{28}$ we re used to calibrate the apparatus. No attempt was made to correct for apparent broadening of the distributions in these analyses. The resulting values of $M_{v^{\prime}}$, the weightaverage molecular weight $M_{w}$, and the number-average molecular weight $M_{n}$ are given in columns $3-5$ of Table I. The GPC values of $M_{v}$ are seen to be in good agreement with the values obtained directly from viscometry, except for the very lowest molecular weight polymer, sample $B$. As shown in the final column of the table, the polydispersity index $M_{w} / M_{n}$ is relatively low for the six polymer fractions, ranging in value from 1.2 to 1.9. The fact that the fractions are not even more nearly monodisperse is apparently due to experimental difficulties in efficiently fractionating polymer chains in this range of molecular weights.

\section{End-linking of the chains}

Networks were prepared from each of the six fractions and from unfactionated sample $A$, using the re$\operatorname{action}^{26}$

$$
\left(\mathrm{C}_{2} \mathrm{H}_{5} \mathrm{O}\right)_{4} \mathrm{Si}+4 \mathrm{HO} \sim \mathrm{OH} \rightarrow{\mathrm{HO} \sim \mathrm{O}^{\prime}}_{\mathrm{HO} \sim \mathrm{O}^{\prime} \sim \mathrm{OH}}^{\mathrm{HO}}+4 \mathrm{C}_{2} \mathrm{H}_{5} \mathrm{OH} \text {, }
$$

where $\mathrm{HO} \sim \mathrm{OH}$ represents the hydroxyl-terminated PDMS chains. (Sample $B$ was excluded because of its very small value of $M_{n}$; it would probably have given a network of insufficient extensibility for reliable stress-elongation measurements.) In the technique employed, $5 \mathrm{~g}$ of the polymer were mixed with $0.3 \mathrm{wt} \%$ of the catalyst stannous-2-ethyl hexanoate, and with exactly the weight $(0.011-0.130 \mathrm{~g})$ of the cross-linking agent tetraethyl orthosilicate which, upon stoichiometric reaction with all of the hydroxyl chain ends, would form a perfect, tetrafunctional network. The mixtures, which appeared to be perfectly homogeneous, were each poured into an aluminum mold of interior dimensions $9 \times 4 \times 0.15 \mathrm{~cm}$. The cross-linking reaction was allowed to proceed under vacuum at room temperature for a total of 2 days. (After the first day, the partially cross-linked sheets were removed from the molds and turned over in order to facilitate removal of the volatile ethanol appearing as a byproduct.) Small portions were cut from three of the completely cured networks and, after extraction with toluene and drying, were tested for unreacted $\mathrm{C}_{2} \mathrm{H}_{5} \mathrm{O}$ groups using the $\mathrm{Zei}-$ 
TABLE II. Elastomeric properties of the end-linked PDMS networks.

\begin{tabular}{lllll}
\hline \hline \multicolumn{5}{c}{ Elongation $^{\mathrm{a}}$} \\
& {$\left[f^{*}\right]_{\text {oqu1 }} /$} & $2 C_{1}$ & $2 C_{9}$ & Swelling \\
Network & {$\left[f^{*}\right]_{\text {ni t }^{\prime}}$} & $\left(N \mathrm{~mm}^{-2}\right)$ & $\left(N \mathrm{~mm}^{-2}\right)$ & $v_{2 m}{ }^{\mathrm{b}}$ \\
\hline$A$ & 0.993 & 0.0892 & 0.0395 & 0.213 \\
$A-1$ & 0.940 & 0.0383 & 0.0297 & 0.153 \\
$A-2$ & 0.983 & 0.0579 & 0.0420 & 0.178 \\
$A-3$ & 0.993 & 0.0843 & 0.0553 & 0.208 \\
$A-4$ & 0.997 & 0.167 & 0.0500 & 0.281 \\
$B-1$ & 1.000 & 0.353 & 0.0313 & 0.368 \\
2 & 1.000 & 0.395 & 0.0205 & 0.381 \\
\hline \hline
\end{tabular}

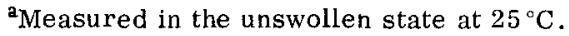

${ }^{b}$ Volume fraction of polymer at equilibrium (maximum) swelling in benzene at room temperature.

sel alkoxy group analysis. ${ }^{28}$ The fact that no $\mathrm{C}_{2} \mathrm{H}_{5} \mathrm{O}$ groups were detected, in conjunction with the known sensitivity of the method, indicates that the desired end-linking reaction went to at least $90 \%$ completion in the case of the largest value of $M_{n}$, and to a considerably higher extent in the case of the others.

\section{Network extraction and swelling equilibrium measurements}

In order to remove the catalyst and any noncrosslinked polymer, each cross-linked sheet was extracted at room temperature, first in tetrahydrof uran for 3 days, and then in toluene for an additional 3 days. The networks, after such extraction, were carefully deswollen in a series of toluene-methanol mixtures of increasing methanol content. The total amount of material thus removed ranged from $1.2 \%$ (network $B-2$ ) to $6.0 \%$ (network $A-1$ ). Swelling measurements ${ }^{29}$ were then carried out on each network in benzene at room temperature, 5 days being allowed for the attainment of equilibrium. The extent of swelling was characterized by $v_{2 m}$, the volume fraction of polymer in the network at equilibrium (maximum) swelling. ${ }^{1}$ It was calculated assuming simple additivity of volumes.

\section{Stress-strain measurements}

Dumbbell-shaped samples having the approximate dimensions $6 \times 0.6 \times 0.1 \mathrm{~cm}$ were cut from each network sheet. Standard techniques ${ }^{30,31}$ were then used to determine stress-strain isotherms for each test sample in the unswollen state, in elongation at $25^{\circ} \mathrm{C}$, under a protective atmosphere of nitrogen. Measurements were made using a sequence of increasing values of the elongation or relative length of the sample $\alpha=L / L_{i}$, with lower values out of sequence frequently investigated to test for reversibility. The value of the force exhibited essentially immediately (within a few seconds) after the deformation was imposed was recorded as its "initial" value. The force was then monitored until it became constant for at least $15 \mathrm{~min}$, which generally required a total time interval of less than $1 \mathrm{~h}$. This constant value of the force was taken to be its "equilibrium" value. Finally, the cross-sectional area $A^{*}$ of each test sample in the unstretched state was measured at room temperature by means of a micrometer.

\section{RESULTS AND DISCUSSION}

The stress-strain data were interpreted in terms of the "reduced stress" defined by $25,30,32,33$

$$
\left[f^{*}\right] \equiv f /\left[A^{*}\left(\alpha-\alpha^{-2}\right)\right] \text {. }
$$

Values of the ratio of the equilibrium value of $\left[f^{*}\right]$ to the initial value exhibited at a particular value of $\alpha$ were very close to unity, as shown by the average values given for this ratio in the second column of Table II. Thus, the se end-linked networks showed very little stress relaxation. The values of $\left[f^{*}\right]$ at equilibrium were plotted as a function of the reciprocal elongation, as suggested by the semiempirical equation of Mooney and Rivlin ${ }^{24}$

$$
\left[f^{*}\right]=2 C_{1}+2 C_{2} \alpha^{-1} \text {, }
$$

in which $2 C_{1}$ and $2 C_{2}$ are constants independent of $\alpha$. The isotherms thus obtained for all of the networks studied a re presented in Fig. 1; the lines shown were located by least-squares analysis. The results obtained upon decreasing $\alpha$ are seen to be in excellent agreement with those obtained from the main sequence of increasing values of $\alpha$, thus demonstrating the reversibility of the stress-strain isotherms. The values of the constants $2 C_{1}$ and $2 C_{2}$ characterizing each isothe rm are given in columns 3 and 4 of Table II.

The elastic modulus, as represented by the constant

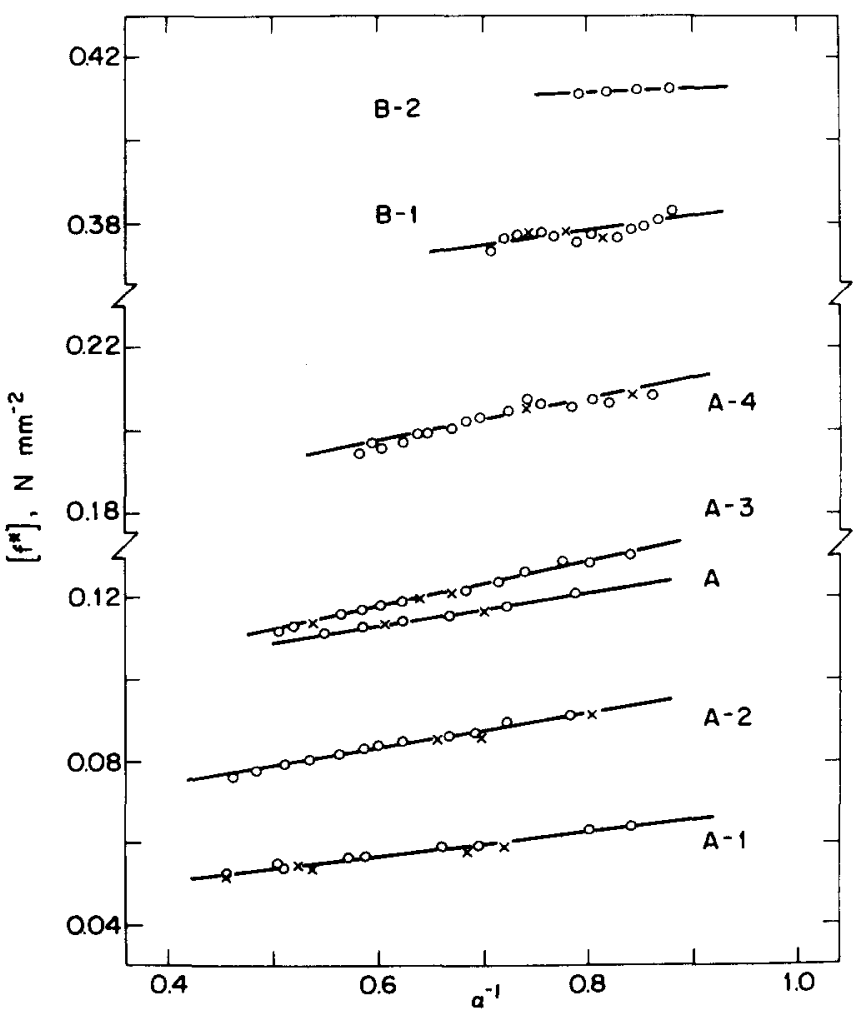

FIG. 1. Stress-strain isotherms for the end-linked PDMS networks in elongation at $25^{\circ} \mathrm{C}$. Results gotten using a series of increasing values of the elongation $\alpha$ are located by the open circles; the other results, obtained using decreasing values of $\alpha$ in order to test for reversibility, are shown by the X's. 


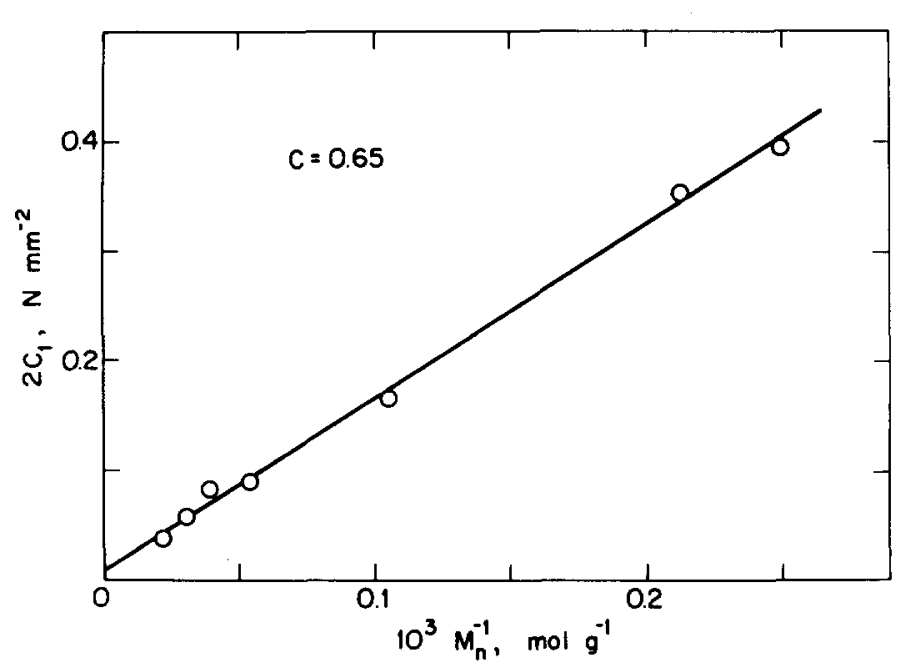

FIG. 2. The elastic modulus, as represented by the constant $2 C_{1}$, shown as a function of the reciprocal of the molecular weight of the chains end-linked to form the network. Interpretation of the results in terms of the theoretical equation ${ }^{17-23}$ $2 C_{1}=C \rho k T M_{n}^{-1}$ gives $C=0.65$.

$2 C_{1}$, was related to the network structure through the equation $1,10,22,23,25$

$$
2 C_{1}=C \rho k T M_{n}^{-1}
$$

derived for networks of Gaussian chains. In this equation $\rho=0.9700 \mathrm{~g} \mathrm{~cm}^{-3}$ is the density of the PDMS networks at $T=298.2 \mathrm{~K}, k$ is the Boltzmann constant, and the molecular weight of the network chains is assumed to be the same as the value of $M_{n}$ of the chains from which it was prepared. In the case of a tetrafunctionally cross-linked network, the constant $C$ is predicted to have the value one-half for the case of a network of "phantom" chains in which the cross-links are free to fluctuate about their mean positions ${ }^{17,19-22}$ and unity for the case in which such fluctuations are assumed to be entirely suppressed, with the crosslinks firmly embedded in the medium by extensive interpretation of chains at the cross-link junctions. ${ }^{22}$ [An additional factor should be introduced into Eq. (5) to take into account the fact that a small amount of the polymer was not successfully incorporated into the network structure, and therefore acted as diluent during the endlinking process. ${ }^{31,34}$ For these networks, however, this factor was found to be within a few percent of unity and has thus been ignored both here and in the analysis of the swelling equilibrium data]. These results, plotted in accordance with Eq. (5), a re presented in Fig. 2. The line shown was located by least-squares analysis; as expected the relationhsip is linear, and the data extrapolate to the origin within experimental error. The slope of the line yields $C=0.65$, which strongly supports the lower part of the range $0.5-1.0$ predicted for this quantity. This is to be expected since the elastic modulus was taken to be $2 C_{1}$, the value of the reduced stress at very high elongation $\left(\alpha^{-1}=0\right)$, where the stresses would be expected to be sufficiently large to overcome constraints imposed on the cross-links by neighboring chain segments. Under these conditions, the cross-links presumably no longer remain firmly embedded in the network medium. The fact that the observed value of $C$ is somewhat larger than the expected value of $0.5^{17,19-22}$ may possibly be due to interchain entanglements (involving segments of two chains relatively remote from their junction points). In any case, these results are quite consistent with those of Allen and co-workers, ${ }^{9,10}$ who report values of the elastic modulus of model networks which are much more consistent with the value of 0.5 , rather than 1.0 , for the coefficient $C$.

The values obtained for the volume fraction $v_{2 m}$ of polymer at swelling equilibrium are given in the last column of Table $\Pi$. They were interpreted using the equation ${ }^{1,2,22,31}$

$M_{n}=-C \rho V_{1}\left(v_{2 m}^{1 / 3}-\omega v_{2 m}\right) /\left[\ln \left(1-v_{2 m}\right)+v_{2 m}+\chi_{1} v_{2 m}^{2}\right]$,

where $\rho=0.9700$ is the density of the PDMS and $V_{1}$ $=89.08 \mathrm{~cm}^{3} \mathrm{~mol}^{-1}$ is the molar volume of the benzene, both at room temperature. Values of the paramete ${ }^{1}$ $\chi_{1}$ characterizing the thermodynamic interactions in the PDMS-benzene system were obtained, as a function of composition, from the work of Flory and Tatara. ${ }^{33}$ The parameter $\omega$ takes into account a possible volumedependent contribution to the free energy of network deformation and, in the case of a tetrafunctionally cross-linked network, should have a value in the range 0 to $\frac{1}{2} .{ }^{22}$ The experimental results, and the theoretical results calculated using $C=0.65$, are shown in Fig. 3 as a function of the molecular weight of the network chains. The circles represent the experimental results, and the solid and dashed lines, the results calculated for $\omega=0$ and $\frac{1}{2}$, respectively. The value $\omega$ $=0$ is seen to give a better representation of the ex-

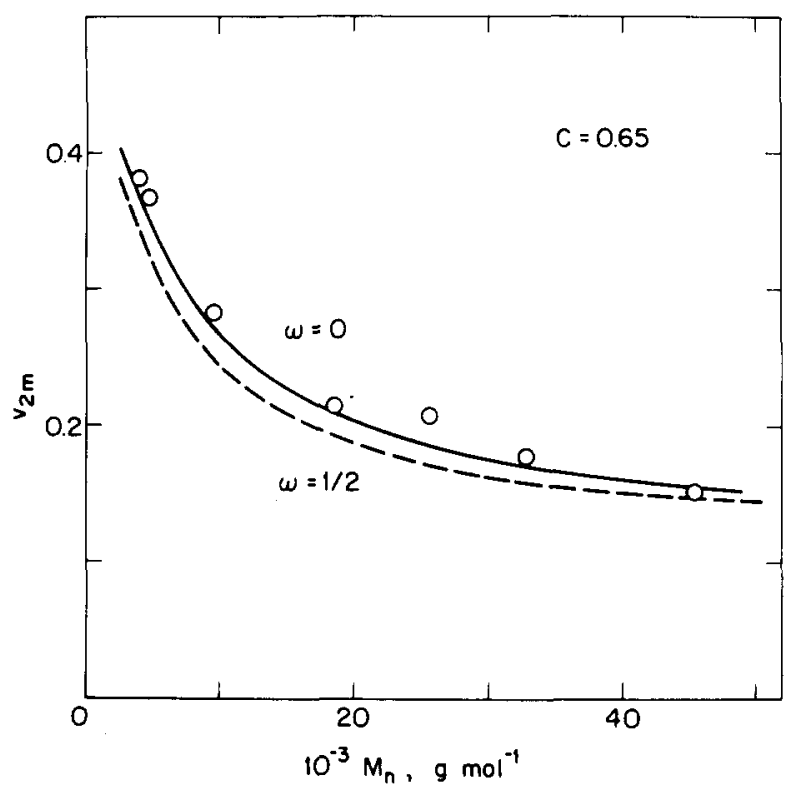

FIG. 3. The volume fraction of polymer at equilibrium (maximum) swelling in benzene at room temperature shows as a function of the molecular weight of the network chains. The lines shown have been calculated from theory $1,2,22,31$ using the result $C=0.65$ established in the preceding figure. The quantity $\omega$ is the coefficient of the logarithmic term in the volume appearing in the expression for the elastic free energy of deformation; its value has been predicted to be in the range $0-\frac{1}{2} .{ }^{22}$ 


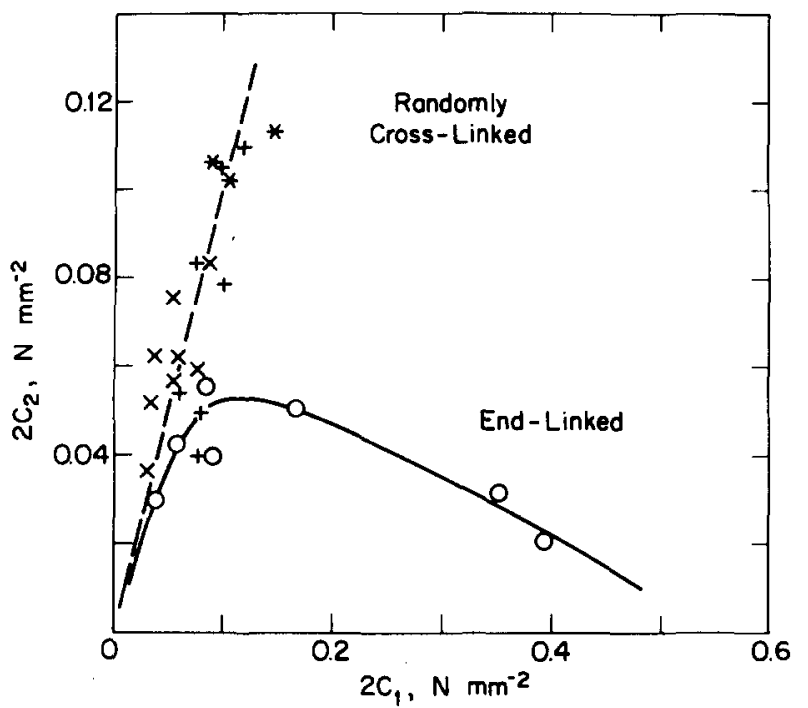

FIG. 4. The Mooney-Rivlin constant $2 C_{2}$ for PDMS networks shown as a function of the degree of cross-linking as measured by $2 C_{1}$. The results for the randomly cross-linked networks are taken from the studies of Mark and Flory ${ }^{25,30}(+)$, Johnson and $\operatorname{Mark}^{25,34}(\mathbf{X})$, and Flory and Tatara ${ }^{33}(*)$. The open circles locate the results obtained in the present study of the endlinked networks.

perimental results, with calculated and experimental values of $v_{2 m}$ agreeing to within ca. $\pm 2 \%$. The alternative value $\omega=\frac{1}{2}$ is, however, also consistent with experiment, the agreement in this case being to within $\pm 5 \%$. As this clearly demonstrates, the extent of swelling of a network prepared in the undiluted state is rather insensitive to this parameter, as has been pointed out previously. ${ }^{31}$ (In fact, $v_{2 m}$ does not vary greatly with changes in either $C$ or $\omega$. For example, at $M_{n}=25000$, the calculated values of $v_{2 m}$ are 0.16 and 0.22 for the extreme values $C=0.50, \omega=\frac{1}{2}$ and $C=1.0, \omega=0$ ). Other studies, ${ }^{7,34,35}$ carried out on networks prepared in solution in order to circumvent this difficulty, are more consistent with the value $\omega$ $=\frac{1}{2}$, and this should probably be considered the more reliable experimental result at the present time. The primary conclusion reached here, therefore, is simply that current theories give a very good account of the equilibrium swelling of these end-linked PDMS networks.

It is of interest to note that all of the elastic moduli are well represented by a single curve, as a re all of the swelling equilibrium results. Since the networks studied had values of the polydispersity index $M_{w} / M_{n}$ ranging from 1.2 to 1.9 , these two elastomeric properties are not greatly dependent on the distribution of chain lengths within the polymer network. Additional experiments covering a wider range of values of $M_{w} /$ $M_{n}$, however, would obviously be of great interest in this regard.

The values of the $2 C_{2}$ constant for the end-linked networks are shown as functions of the elastic modulus, as represented by $2 C_{1}$, in Fig. 4 . Included for purposes of comparison are corresponding results ${ }^{25,30,33,34}$ obtained for randomly cross-linked PDMS networks which were also prepared in the undiluted state and studied unswollen. The end-linked PDMS networks are seen to have very small values of $2 C_{2}$ at large $2 C_{1}$; similarly small values of $2 C_{2}$ have been reported for end-linked networks of polybutadiene ${ }^{6}$ and of polyisoprene ${ }^{16}$ in this range of values of $2 C_{1}$. Since the constant $2 C_{2}$ has been widely attributed to interchain entanglements, it was this observation which led to the suggestion ${ }^{6,16}$ that end-linked networks may be essentially free of such entanglements. End-linked networks are not unusual, however, in regard to their having very small values of $2 C_{2}$ at large $2 C_{1}$. Unfortunately, in the studies of the randomly cross-linked PDMS networks, obtained by the high energy irradiation of very long PDMS chains, networks of the required degree of extensibility could not be prepared at such very high values of $2 C_{1},{ }^{30}$ suggesting that this type of cross-linking gives an unusually heterogeneous distribution of cross-links. (In the case of the end-linked PDMS networks, those having the highest values of $2 C_{1}$ are, in fact, unusually extensible, considering their very high degree of cross-linking. This may be due to their relatively small values of $M_{w} / M_{n}$, and the associated absence of very short network chains.) It is quite relevant, however, that in the case of natural rubber, one of the very few randomly cross-linked polymers which have been

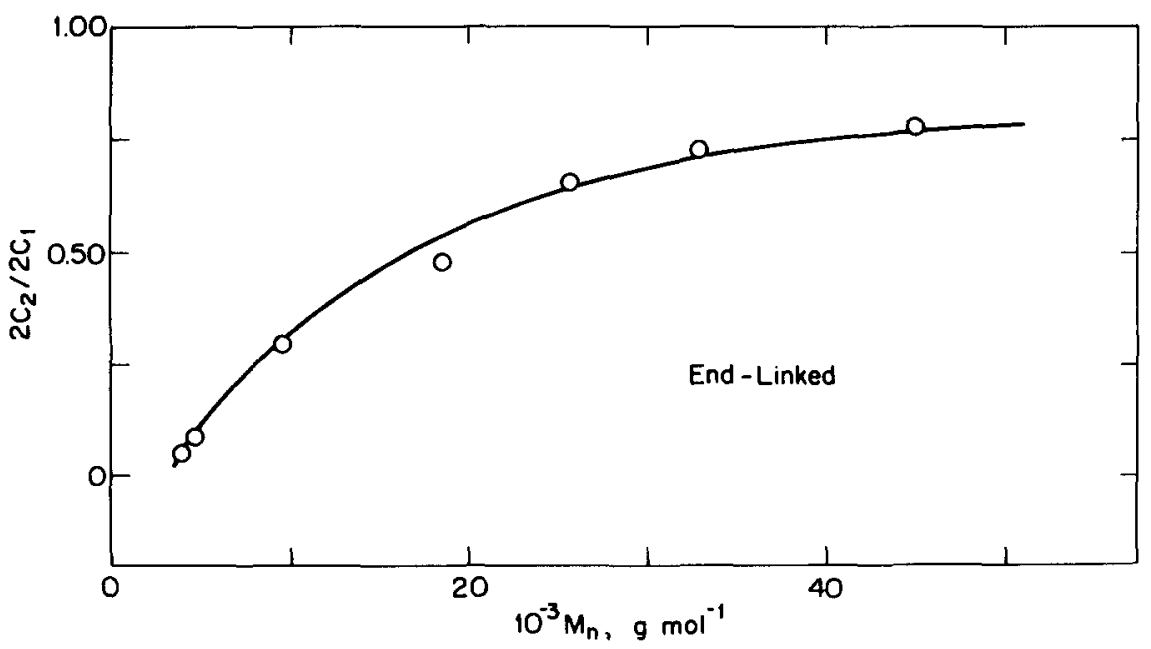

FIG. 5. The constant $2 C_{2}$, approximately normalized to constant cross-link density, for end-linked PDMS networks; the results are shown as a function of the molecular weight of the chains used to form the network. 
studied at such high degrees of cross-linking, $2 C_{2}$ is again very small in this region of large $2 C_{1}$. Also, it is dif ficult to visualize how chains of the lengths studied here would become disentangled in any end-linking process. In fact, comparison of the end-to-end distance of such chains with the average distance between cross-links suggests that there should be some interpenetration. Finally, as mentioned earlier, the value of the coefficient $C$ determined from the values of the elastic modulus is itself suggestive of the presence of some entanglements in these end-linked networks. The number of such entanglements in these end-linked networks may be relatively small, but such may also be the case in the usual types of networks, i. e., those prepared by the random cross-linking of much longer polymer chains. ${ }^{22}$

Values of the ratio $2 C_{2} / 2 C_{1}$, which represents the constant $2 C_{2}$ approximately normalized to constant cross-link density, ${ }^{25}$ are shown as a function of the network chain length in Fig. 5. The ratio is seen to decrease monotonically with decrease in the average length of the network chains. A recent theoretical estimate $^{23}$ of the dependence of $2 C_{2} / 2 C_{1}$ on degree of cross-linking seems to be consistent with this observation.

\section{ACKNOWLEDGMENTS}

It is a pleasure to acknowledge financial support from the National Science Foundation, and several very helpful discussions with Professor P. J. Flory of Stanford University.

${ }^{1} \mathrm{P}$. J. Flory, Principles of Polymer Chemistry (Cornell University Press, Ithaca, N. Y., 1953).

${ }^{2}$ L. R. G. Treloar, The Physics of Rubber Ela sticity (Clarendon Press, Oxford, 1958).

${ }^{3}$ K. Dušek and W. Prins, Adv. Polymer Sci. 6, 1 (1969).

${ }^{4}$ W. W. Graessley, Adv. Polymer Sci. 16, 1 (1974).

${ }^{5}$ C. A. Uraneck, H. L. Hsieh, and O. G. Buck, J. Polymer Sci. 46, 535 (1960).

${ }^{6}$ G. Kraus and G. A. Moczvgemba, J. Polymer Sci. A 2, 277 (1964).

${ }^{7}$ D. Froelich, D. Crawford, T. Rozek, and W. Prins, Macromolecules 5, 100 (1972).
${ }^{8}$ G. Beinert, A. Belkebir-Mrani, J. Herz, G. Hild, and P. Rempp, Faraday Disc. Chem. Soc. 57, 27 (1974).

${ }^{9}$ D. J. Walsh, G. Allen, and G. Ballard, Polymer 15, 366 (1974).

${ }^{10}$ G. Allen, P. A. Holmes, and D. J. Walsh, Faraday Disc. Chem. Soc. 57, 19 (1974); G. Allen, P. L. Egerton, and D. J. Walsh, Polymer 17, 65 (1976).

${ }^{11} \mathrm{P}$. Rempp, in Reactions of Polymers, edited by J. A. Moore (Reidel, Boston, 1973); and pertinent references cited therein.

${ }^{12}$ F. Rietsch and D. Froelich, Polymer 16, 873 (1975).

${ }^{13}$ P. Rempp, J. Herz, G. Hild, and C. Picot, Pure Appl. Chem. 43, 77 (1975); and pertinent references cited the rein.

${ }^{14}$ J. P. Munch, S. Candau, R. Duplessix, C. Picot, J. Herz, and H. Benoit, J. Polymer Sci. Polymer Phys. Ed. 14, 1097 (1976).

${ }^{15}$ M. Morton, L. J. Fetters, J. Inomata, D. C. Rubio, and R. N. Young, Rubber Chem. Technol. 49, 303 (1976).

${ }^{16} \mathrm{M}$. Morton and D. C. Rubio (in press); paper no. 43, Cleveland Meeting, Rubber Div., ACS, 1975.

${ }^{17}$ H. M. James and E. Guth, J. Chem. Phys. 15, 669 (1947).

${ }^{18}$ P. J. Flory, Trans. Faraday Soc. 57, 829 (1961).

${ }^{19}$ B. E. Eichinger, Macromolecules 5, 496 (1972).

${ }^{20}$ W. W. Graessley, Macromolecules 8, 186, 865 (1975).

${ }^{21}$ R. T. Deam and S. F. Edwards, Phil. Trans. R. Soc. Lond. Ser. A $280,317(1976)$

${ }^{22}$ P. J. Flory, Disc. R. Soc. Lond. (in press).

${ }^{23}$ P. J. Flory (in preparation).

${ }^{24}$ M. Mooney, J. Appl. Phys. 19, 434 (1948); R. S. Rivlin, Phil. Trans. Roy. Soc. London, Ser. A 241, 379 (1948).

${ }^{25}$ J. E. Mark, Rubber Chem. Technol. 48, 495 (1975).

${ }^{26}$ We are indebted to John Saam of the Dow Corning Corporation for the two samples of hydroxyl-terminated PDMS (designated E-1586-148A and 148B, respectively), for a great deal of helpful advice on end-linking them and for several standard PDMS samples suitable for calibrating our gel permeation chromatography apparatus.

${ }^{27}$ A. J. Barry, J. Appl. Phys. 17, 1020 (1946).

${ }^{28}$ Analyses for $\mathrm{C}_{2} \mathrm{H}_{5} \mathrm{O}$ groups were carried out by the Schwartzkopf Microanalytical Lab., Woodside, N. Y.

${ }^{29}$ J. E. Mark, J. Phys. Chem. 68, 1092 (1964).

${ }^{30}$ J. E. Mark and P. J. Flory, J. Appl. Phys. 37, 4635 (1966).

${ }^{31}$ J. E. Mark, J. Am. Chem. Soc. 92, 7252 (1970).

${ }^{32}$ A. Ciferri and P. J. Flory, J. Appl. Phys. 30, 1498 (1959).

${ }^{33}$ P. J. Flory and Y. Tatara, J. Polymer Sci., Polymer Phys. Ed. 13, 683 (1975).

${ }^{34}$ R. M. Johnson and J. E. Mark, Macromolecules 5, 41 (1972).

${ }^{35} \mathrm{C}$. U. Yu and J. E. Mark, Polymer J. 7, 101 (1975). 